Revista Verde de Agroecologia e Desenvolvimento Sustentável

http://www.gvaa.com.br/revista/index.php/RVADS

ARTIGO CIENTÍFICO

[BSW १९९१-8208

\title{
Estudo cinético da secagem da uva Isabel para produção de uva passa
}

\author{
Kinetic study of the drying of the grape Isabel for grape production it passes.
}

\author{
Antônio Vitor Machado ${ }^{1}$, José Aldenor de Souza ${ }^{2}$, Rafael da Silva Novaes ${ }^{3}$
}

\begin{abstract}
Resumo- A fruticultura é um dos segmentos mais importantes na agricultura nacional, porém um grande problema enfrentado pelos fruticultores é a conservação dos frutos maduros. A falta de tecnologias adequadas para o melhor aproveitamento dos frutos tem resultado em um alto índice de desperdício, cerca de toneladas ano. A uva Isabel, é uma das principais variedades Vitis labrusca, que apresenta sabor característico e pigmentação bastante acentuada, destacando-se como uva de mesa comum, apresentando-se como uma variedade de grande aceitação pelo consumidor e pela indústria de alimentos. A desidratação de frutas vem sendo objeto de muitas pesquisas com o propósito de se obter técnicas que proporcionem, além de baixo custo de produção, a manutenção da qualidade dos produtos desidratados e a redução das perdas pós-colheita. Neste sentido o presente trabalho objetivou estudar a secagem da uva Isabel para obtenção de uva passa desidratada, visando obter informações sobre a qualidade do fruto in-natura e após processamento, realizando-se para isto à caracterização físico-química do fruto in-natura e desidratado. Foram realizadas análises de pH, sólidos solúveis, acidez total titulável, umidade, atividade de água e do teor de antocianinas nos Laboratórios de Tecnologia da UFERSA e UFCG. De acordo com os resultados obtidos, os frutos desidratados apresentaram um incremento nos teores de sólidos solúveis (de 11,17 para $45^{\circ}$ brix), da acidez titulável (de 0,73 para $1,34 \%$ ) e do teor de antocianina, que foi elevado de $117,83 \mathrm{mg} / 100 \mathrm{~g}$ para $380,52 \mathrm{mg} / 100 \mathrm{~g}$ de fruto desidratado, e redução quanto aos valores de umidade (de 85,65 para 21,75\%) e atividade de água (de 0,90 para 0,66), estes valores de nutrientes apresentaram-se próximos aos relatados na literatura demonstrando que a secagem solar é uma excelente alternativa para a conservação da uva Isabel, contribuindo assim para redução das perdas pós-colheita e com a agregação de valor ao produto desidratado.
\end{abstract}

Palavras chave: Cinética, secagem, uva passa.

\begin{abstract}
The fruticultura is one of the segments most important in national agriculture, however a great problem faced for the fruticultores is the conservation of the mature fruits. The lack of technologies adjusted for optimum exploitation of the fruits has resulted in one high index of wastefulness, about tons year. The grape Isabel, is one of the main Vitis labrusca varieties, that it presents characteristic flavor and pigmentação sufficiently accented, being distinguished as grape of common table, presenting itself as a variety of great acceptance for the consumer and the food industry. The dehydration of fruits comes being object of many research with the intention of if getting techniques that they provide, beyond low cost of production, the maintenance of the dehydrated product quality and the reduction of the losses after-harvest. In this direction the present work objectified to study the drying of the grape Isabel for grape attainment passes dehydrated, aiming at to get information on the quality of the fruit in-natura and after processing, becoming fullfilled itself for this to the characterization physicist-chemistry of the fruit dehydrated and in-natura. Soluble analyses of $\mathrm{pH}$, solids, titulável total acidity, humidity, activity of water and the text of antocianinas in the Laboratories of Technology of UFERSA and UFCG had been carried through. In accordance with the gotten results, the dehydrated fruits had presented an increment in texts of soluble solids (of 11,17 for $45^{\circ}$ brix), of the titulável acidity (of 0,73 for $1,34 \%$ ) and of the text of antocianina, that was raised of $117,83 \mathrm{mg} / 100 \mathrm{~g}$ for $380,52 \mathrm{mg} / 100 \mathrm{~g}$ of dehydrated fruit, and reduction how much to the values of humidity (of 85,65 for 21,75\%) and activity of water (of 0,90 for $0,66)$, these values of nutrients had been presented next to the told ones in literature demonstrating that the solar drying is an excellent alternative for the conservation of the grape Isabel, thus contributing for reduction of the losses after-harvest and with aggregation of value to the dehydrated product.
\end{abstract}

Keywords: Kinetic, drying, grape pass.

\footnotetext{
*Autor para correspondência

Recebido para publicação em 22/02/2015; aprovado em 30/02/2015

${ }^{1}$ Professor adjunto UFERSA E-mail: machadoav@ufersa.edu.br

${ }^{2}$ Mestre em Sistemas Agroindustriais, PPGSA-UFCG, Pombal-PB. E-mail: aldenora@hotmail.com

${ }^{3}$ Aluno mestrado em Sistemas Agroindustriais, UFCG, Pombal-PB. E-mail: rafaelnovaes@ @otmail.com
} 


\section{INTRODUÇÃO}

A fruticultura é, hoje, um dos segmentos de maior importância da agricultura nacional respondendo por mais de $40 \%$ da produção agrícola. As frutas são de grande importância em todo o mundo, no que se refere aos aspectos social, econômico e alimentar. A fruticultura possibilita a exploração intensiva de áreas produtivas, tornando-as lucrativas. Além disso, utiliza elevada quantidade de mão-deobra, constituindo-se numa fonte geradora de empregos não somente na produção, como também no armazenamento, no processamento e na comercialização de frutas (Machado, 2014).

O Brasil é o terceiro maior produtor mundial de frutas, com uma produção que supera 44 milhões de toneladas em 2014. A base agrícola da cadeia produtiva das frutas abrange mais de 2,2 milhões de hectares, gera mais de 4 milhões de empregos diretos e indiretos, além disso, para cada 10.000 dólares investidos em fruticultura, são gerados 3 empregos diretos permanentes e dois empregos indiretos (IBRAF, 2013).

Nos últimos anos, a produção de frutas cresceu a uma taxa nunca vista antes na história, entre 2002 e 2008, a produção nacional cresceu $28 \%$, saindo de 34 milhões de toneladas em 2001 para mais de 44 milhões de toneladas em 2014. O potencial de expansão para os próximos quatro ou cinco anos é de 4,5\% ao ano (LACERDA et al., 2014).

A região Nordeste vem se destacando na produção de frutas como o abacaxi, melão, uva, banana, manga e caju, pois as condições climatológicas são muito mais favoráveis do que nas regiões sul e sudeste do Brasil (TODAFRUTA, 2013).

A maioria dos frutos tropicais como o abacaxi, a uva, a banana, a manga e o caju apresentam alto grau de perecibilidade e elevados índices de perdas pós-colheita, motivo este foco de diversas pesquisas na área de processamento e conservação destes, entre as várias formas de conservação estudadas está à desidratação ou secagem, o processamento mínimo, o congelamento, entre outras (EMEPA, 2013).

Os frutos tropicais compartilham algumas características que os tornam inconfundíveis em sua constituição, como grande diversidade de vitaminas, carboidratos e minerais. Além do sabor e odor agradável, possuindo elevada aceitabilidade e também inúmeros componentes bioativos de grande importância para uma vida saudável (MACHADO, 2012).

A uva Isabel, é uma das principais variedades Vitis labrusca, é originária do sul dos Estados Unidos, cultivada principalmente nas regiões sul e sudeste do Brasil, nos últimos anos vem se destacando também na região nordeste. É a principal cultivar dos vinhedos do Rio Grande do Sul e de Santa Catarina, já sendo cultivada também com sucesso no Mato Grosso, em Goiás e em Pernambuco e na Zona da Mata. Demonstrou boa adaptação no Noroeste de São Paulo e no Triângulo Mineiro (MAIA et al., 2002) e vem sendo plantada em projetos recentes voltados à produção de uvas para suco no Vale do Submédio São Francisco. No Pólo Agroindustrial de Petrolina-Juazeiro, onde se encontram grandes produtores de uva do país, tendo já alcançado mercados externos na atualidade.
O predomínio da uva 'Isabel' nas regiões tradicionais, assim como sua expansão em novos pólos, decorre de sua facilidade de adaptação às diferentes condições ambientais e à qualidade da uva, originando produtos com tipicidade e de boa aceitação no mercado (CAMARGO, 2010).

A uva 'Isabel' é uma variedade rústica, menos exigente a tratos culturais e, por ser mais tolerante às doenças fúngicas, é bem adaptada às condições de clima úmido, possui sabor característico das labruscas, adaptando-se a todos os usos, é consumida como uva de mesa, usada para a elaboração de vinhos brancos, rosados e tintos, os quais, muitas vezes, são utilizados para a destilação ou para a elaboração de vinagre. Além de originar suco de boa qualidade, pode ser matéria prima para doces, geleia entre outros produtos (DETONI et al., 2005).

Devido ao seu aroma característico, que mascara a percepção de aromas refinados do vinho, seu uso é limitado para elaboração de vinhos finos. A uva Isabel, apresenta sabor característico e pigmentação bastante acentuada, tornando esta variedade de uva bastante apreciada para a indústria de alimentos (TODAFRUTA, 2010).

Um grande problema enfrentado pelos fruticultores é a conservação dos frutos maduros, motivo este, responsável pela perda de grande parte da colheita, estimasse uma percentagem de $40 \%$ de perdas entre a colheita no campo até a chegada ao mercado consumidor final. Este alto índice de desperdício é responsável por grandes aumentos de preço das frutas para o consumidor final. Uma das principais causas de perdas é a deterioração, devido à alta quantidade de água livre presente nos frutos. Portanto, faz se necessário à utilização de métodos de conservação a fim de que o alimento se torne estável à deterioração química e microbiana, segundo ALVES et al., (2011, MACHADO, 2014).

A desidratação de frutas é um mercado promissor e com grande potencial de crescimento e muito pouco explorado empresarialmente ainda no Brasil (MACHADO, 2009). A desidratação de alimentos sólidos, como frutas e hortaliças, normalmente significa remoção da umidade de sólido por evaporação, e tem por objetivo assegurar a conservação das frutas por meio da redução do seu teor de água. Essa redução deve ser efetuada até um ponto, onde a concentração de açúcares, ácidos, sais e outros componentes seja suficientemente elevada para reduzir a atividade de água, inibindo, portanto, o desenvolvimento de microrganismos. Deve-se ainda conferir ao produto final características sensoriais próprias e preservar ao máximo o seu valor nutricional (MACHADO, 2012).

Entre as principais vantagens oferecidas pela secagem de frutas está a concentração dos nutrientes e o maior tempo de vida de prateleira. Além disso, o sabor permanece quase inalterado por longo tempo, uma vez que é minimizada a proliferação de microorganismos, devido a redução da atividade de água do produto.

A secagem é atualmente empregada não apenas com o objetivo de conservação dos alimentos, mas também para elaboração de produtos diferenciados, como por exemplo, as massas, biscoitos, farinhas, iogurtes, sorvetes entre outros (FIOREZE, 2004).

As frutas tropicais apresentam características peculiares a uva, assim como outras frutas, apresentam curto período de comercialização pós-colheita, consequentemente 
são necessários estudos e pesquisas sobre a sua desidratação. Desta forma o presente trabalho objetivou a secagem da uva Isabel da variedades Vitis labrusca, para a obtenção de uva passa desidratada, visando também obter informações sobre a qualidade do fruto in-natura e após processamento, realizando à caracterização físico-química do fruto in-natura e desidratado. Buscando assim a viabilização da secagem como uma tecnologia viável para conservação desta fruta.

\section{MATERIAL E MÉTODOS}

A matéria prima utilizada foi uva Isabel da variedades Vitis labrusca, adquiridas no comércio de Caraúbas - RN . Os frutos foram selecionados de acordo com a coloração (vermelha escura) da casca, de forma ovóide e ausência de danos físicos (injúrias), com o grau de maturidade comercial adequado para o processamento.

Em seguida os frutos foram levados para o laboratório de Laboratório de Tecnologia da UFERSA e da UFCG para processamento e caracterização físico-química.

O processamento iniciou-se com a sanitização com água contendo $20 \mathrm{ppm}$ de cloro livre, sendo os cachos despencados e as uvas dispostas em bandejas de aço inox perfuradas, sendo estas levadas para secagem.

A secagem dos frutos foi realizada em estufa de circulação de ar forçado com controle da temperatura do ar de secagem a $60^{\circ} \mathrm{C}$, onde as bandejas foram pesadas em intervalos de tempo regulares de 30 em 30 minutos, até atingir peso constante.

A caracterização físico-química do fruto in-natura e após desidratado, foi realizada conforme as normas e os procedimentos do INSTITUTO ADOLFO LUTZ (2008), descritos a seguir: Sólidos solúveis, determinado no filtrado por refratometria, utilizando-se refratômetro digital, e os resultados expressos em ${ }^{\circ} \mathrm{Brix} ; \mathrm{pH}$, determinado no filtrado diretamente, utilizando-se pHmetro digital digimed mod- 39, acidez total titulável, obtida por titulação do filtrado com $\mathrm{NaOH} 0,1 \mathrm{~N}$, e expressa em porcentagem de ácido tártarico; a determinação da umidade foi realizada em estufa de circulação de ar (mod. ORION, marca FANEM), á temperatura de $105^{\circ} \mathrm{C}$ por 24 horas, a atividade de água foi medida utilizando o aparelho portátil denominado Decagon de marca Braseq, modelo Pawkit, com faixa de medição de (Aw) de 0,00 a 1,00, para o teor de antocianinas foi utilizado o espectrofotômetro B442 (Marca MICRONAL), em $\lambda=$ $520 \mathrm{~nm}$.

$\mathrm{O}$ experimento foi realizado em delineamento inteiramente casualisado (DIC), com um mínimo de três repetições. Os dados foram analisados utilizando-se o programa Sistema para Análise de Variância (SISVAR), e as médias comparadas através do teste de Tukey (5\%) de probabilidade (FERREIRA, 2010), as curvas de secagem foram submetidas à análise de variância estudando seus valores em função do tempo de secagem através da análise de regressão.

\section{RESULTADOS E DISCUSSÃO}

De acordo com os dados apresentados na Tabela 1, sobre a caracterização físico-química, da uva Isabel da variedade Vitis labrusca, para o fruto in-natura e desidratado, pode-se notar que os valores médios de sólidos solúveis totais para o fruto in-natura foi de $12,09^{\circ}$ brix, valor este próximo aos relatados por (CORRALES, 2010), Ribeiro et. al. (2009) e Abel (2007), os quais relataram teores de sólidos solúveis de 13,0 a 14,5 brix para as variedade de uvas Benitaka, Vitis labrusca. e Vitis vinifera L. O teor de sólidos solúveis determinam a doçura do fruto durante a maturação e está diretamente relacionada ao seu sabor e aceitação (KAWAMATA, 1997). Após processamento a uva desidratada, demonstrou um aumento significativo do teor de açucares para $46,38{ }^{\circ}$ Brix, isto se deve principalmente à perda de água pelo fruto, tendo como principal consequência a concentração de seus nutrientes.

A uva Isabel da variedade Vitis labrusca, apresentou para o fruto in-natura uma acidez titulável de $0,76 \mathrm{~g}$ de ácido tartárico/100 $\mathrm{mL}$ de suco, valor este próximo aos $(0,8 \mathrm{~g}$ de ácido tartárico/100 mL de suco), relatados por Melo, (2011), Bouhamidi, (2009), e Santana et. al. (2008), para as variedade de uva Patrícia e Vitis vinifera $L$. As variações da acidez titulável estão relacionadas às variações climáticas principalmente com a elevação da temperatura na época de maturação dos cachos de uva, tipo de solo, técnicas de manejo do parreiral e região de cultivo.

O fruto após desidratado apresentou um teor médio de 1,31\% de ácido tartárico/100 mL de suco, valor este característico para este tipo de fruto, conforme o grau de maturação do mesmos, segundo relatos de Carvalho \& Chitarra (1984), para uva in natura teores abaixo de $1,5 \%$ apresentam-se como adequados para este fruto.

Os valores médios de umidade para o fruto innatura e após desidratados oscilaram entre $87,46 \%$ para $19,87 \%$ (bu), respectivamente, o que implicou uma redução de $77,2 \%$ do teor de água presente no fruto in-natura para a uva desidratada após processamento, estes valores demonstram-se coerentes aos encontrados na literatura, conforme relatados por MACHADO (2011), THÉ (2010), (CHAUCA, 2004) em seus experimentos de secagem de frutos.

A atividade de água (Aw) é um fator intrínseco ao alimento, que demonstra a quantidade de água livre neste, disponível, ou seja conteudo este responsável pelo crescimento de microrganismos e para reações que possam deteriorar e inutilizar o mesmo para o consumo. Sua quantificação é de fundamental importância para averiguar a estabilidade do mesmo após processamento. O teor médio de atividade de água (Aw) detectado foi 0,89 para o fruto innatura, passando para o teor 0,60 de atividade de água (Aw), demonstrando assim uma boa estabilidade para a uva após processamento.

A uva Isabel da variedade de Vitis labrusca apresentou um teor médio de antocianinas de 118,84 $\mathrm{mg} / 100 \mathrm{~g}$, para a fruta in-natura e de $378,95 \mathrm{mg} / 100 \mathrm{~g}$ para a fruta desidratada. De acordo com a literatura o teor de antocianinas encontrado em uvas tintas variam de 30 a 750 $\mathrm{mg} / 100 \mathrm{~g}$ de fruto maduro, segundo Malacrida (2006), o teor de antocianinas presentes nas uvas diferem de acordo com a espécie, cultivar, maturidade, condições climáticas, entre outros fatores (MATTIVI et al., 2006; MUÑOZ-ESPADA et al., 2004 e MAZZA, 1995).

Os resultados obtidos a partir dos experimentos de secagem com o secador convencional de bandejas, estão apresentados na Figura 1, onde visualiza-se a curva cinética de secagem da uva Isabel da variedades Vitis labrusca, na forma adimensional pela razão de umidade, U*, versus tempo de processamento. 
A curva de secagem apresenta no inicio do processo um período de aquecimento bem acentuado, seguido de um período de taxa constante e um período de taxa decrescente até um tempo de 400 minutos indicando o fim do processamento para esta variedade de uva. A curva demonstra-se como típica para secagem de frutos.

Tabela 1. Valores médios da caracterização físico-químicas da uva Isabel “in natura" e após desidratada.

\begin{tabular}{ccc}
\hline Determinações & "in natura" & Desidratada \\
\hline Atividade de água (Aw) & 0,89 & 0,60 \\
Umidade (\% bu) & 87,46 & 19,87 \\
Antocianinas (mg/100g) & 118,84 & 378,95 \\
Acidez Total Titulável & 0,76 & 1,31 \\
$(\%$ ácido tartárico/100 mL $)$ & & 4,13 \\
pH & 3,51 & 46,38 \\
Sólidos Solúveis $\left({ }^{\circ}\right.$ Brix $)$ & 12,09 & \\
\hline
\end{tabular}

Figura 1. Curva cinética da secagem da uva Isabel, pela razão de umidade, U*, versus tempo de operação.

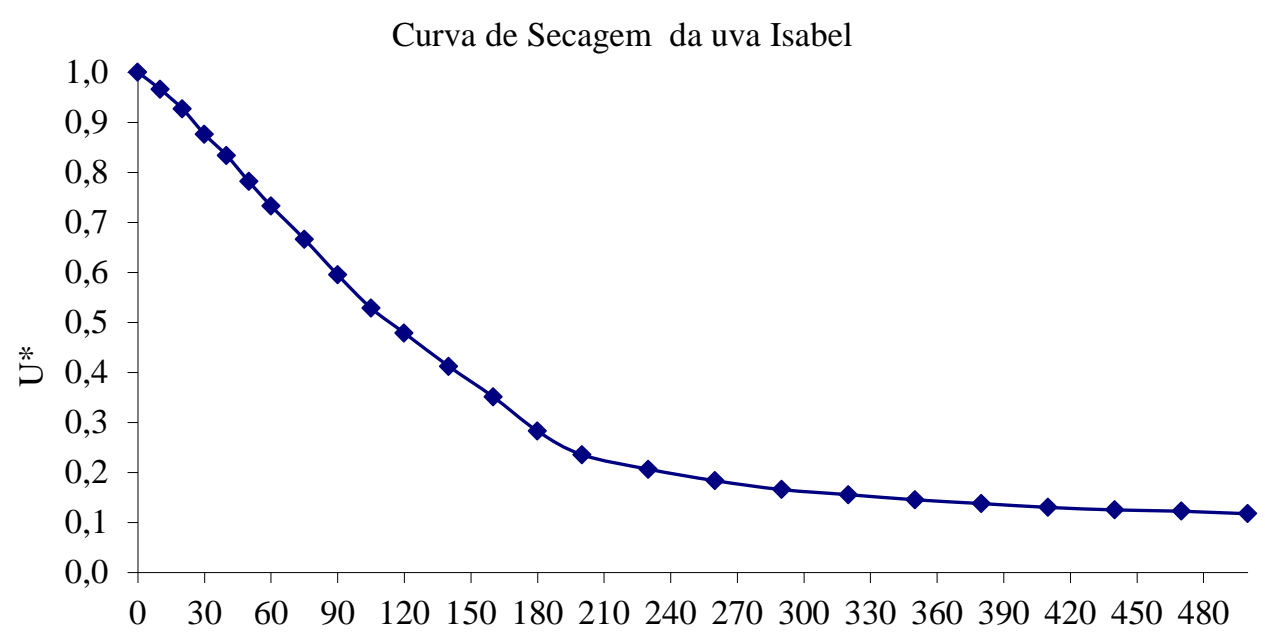

Tempo(min)

\section{CONCLUSÕES}

A caracterização físico-química, da uva Isabel da variedade Vitis labrusca, in natura, apresentou um aumento nos teores de ${ }^{\circ}$ Brix, $\mathrm{pH}$, acidez titulável e antocianinas, quando comparadas a uva desidratada, isto se deve a uma maior concentração desses constituintes em consequência da perda de água do fruto. Quanto as variáveis umidade e atividade de água observou-se uma diminuição em seus teores para o fruto seco, relacionado com o processo da desidratação aplicado na matéria-prima.

A uva Isabel da variedades Vitis labrusca apresenta um grande potencial para industrialização através da desidratação, demonstrando que a secagem é uma excelente alternativa para a conservação da uva Isabel, contribuindo assim para redução das perdas pós-colheita e com a agregação de valor ao produto desidratado.

\section{REFERÊNCIAS BIBLIOGRÁFICAS}

ABE, L. T.; DA MOTA, R. V.; LAJOLO, F. M.; GENOVESE, M. I. Compostos fenólicos e capacidade antioxidante de cultivares de uvas Vitis labrusca L. e Vitis vinifera L. Ciências e Tecnologia de Alimentos. Campinas, 27(2), p. 394-400, abr.-jun. 2007.
ARAÚJO, J. Como fazer farinha de uva. 2010, disponível em:

<http://blog.jarioaraujo.com/2010/nutricao/143/comofazer-farinha-de-uva/>. Acesso em: 17 nov. 2012.

ARVANITOYANNIS, I. S.; LADAS, D.; MAVROMATIS, A. Potential uses and applications of treated wine waste: a review. International Journal of Food Science and Technology, v. 41, p. 475-487, 2006.

BARBETTA, P. A. Estatística aplicada às Ciências Sociais. 4 ed.Florianópolis: UFSC, 2001. 338p.

BOUHAMIDI, R.; PRÉVOST, V.; NOUVELOT, A. High protection by grape seed proanthocyanidins (GSPC) of polyunsaturated fatty acids against UV-C induced peroxidation. Comptes rendus de l'Académie des sciences. Série III, Sciences de la vie, Montrouge, v. 321, p. 31-38, 1998.

CAMARGO, U. A. 'Isabel Precoce': Alternativa para a Vitivinicultura Brasileira. Bento Gonçalves: Embrapa Uva e Vinho, 2004. 6p. (Embrapa Uva e Vinho. Comunicado Técnico, 54).

CANO-CHAUCA, M. N., Indução de cristalização de açúcares de frutos tropicais em pó obtidos por spray 
drying e sua caracterização funcional. Tese de Doutorado em Ciências dos Alimentos UFLA LavrasMG, 2004.

CORRALES, M.; FERNANDEZ, A.; PINTO, M. G. V.; BUTZ, P.; FRANZ, C. M. A. P.; SCHUELE, E.; TAUSCHER, B.; Characterization of phenolic content, in vitro biological activity, and pesticide loads of extracts from white grape skins from organic and conventional cultivars. Food and Chemical Toxicology. V. 48, p. 3471-3476, Dez. 2010.

CARVAlHO, V. D.; CHITARRA, M. I. F. Aspectos quantitativos da uva. Informe Agropecuário, Belo Horizonte, v. 10, n. 117, p. 75-79, 1984.

DETONI, A. M.; ClEMENTE, E.; BRAGA, G. C.; HERZOG, N. F. M. Uva Niágara Rosada cultivada no sistema orgânico e armazenada em diferentes temperaturas. Ciência Tecnologia Alimentos, Campinas, v. 25, n.3, p. 546-552, 2005.

EMEPA. Uva < http://www.emepa.org.br/sigatoka_.php $>$ Data da Edição 25 de março 2009. Acesso em $15 / 04 / 2013$.

FIOREZE, R. Principios de secagem de produtos biologicos, Joao Pessoa. Editora Universitaria - UFPB, p.229, 2004.

FERREIRA, A.B.H. Desidratacao. In: Novo dicionario da lingua portuguesa. Rio de Janeiro: Nova Fronteira S.A., p.565, 2003.

http://www.todafruta.com.br/todafruta/mostra_conteudo.asp ? Acesso em 11/05/2013. Data da Edição: 25/01/09 Fonte: Núcleo de Estudo - Instituto Brasileiro de Fruticultura - IBRAF.

KAWAMATA, S. Studies on sugar component for fruits by gas-liquid chromatography. Bulletin Tokio Agricultural Experiment Station, Tokio, n. 10, p. 5363, 1997. In: RIBEIRO, S.; MATOS, G.; MARQUES, M.; LIMA, A. Caracterização físico-química, fenólicos totais e capacidade antioxidante de uvas Benitaka cultivadas no estado do Piauí-brasil. IV Congresso de Pesquisa e Inovação da Rede Norte e Nordeste de Educação Tecnológica. 4.2009, Belém. Anais... Belém: IF - Belém, 2009.

MACHADO, A.V.; BARBOSA, L.S; MACEDO, J.L.; SANTOS, C.M. Estudo da secagem de frutos tropicais do Nordeste. Revista Verde de Agroecologia e Desenvolvimento Sustentável, Mossoró - RN, v.9, n.1, p.186-190, jan-mar, 2014.

MACHADO, A.V.; ALVES, F.M.S.; QUEIROGA, K.H. Alimentos produzidos a partir de farinha de caju, obtida por secagem Revista Verde de Agroecologia e
Desenvolvimento Sustentável, Mossoró - RN, v.6, n.3, p.131-138, julho/setembro, 2012. v.6,

MACHADO, A.V.; OLIVEIRA, E.L.; SANTOS, E.S.; OLIVEIRA, J.A. Influência da espessura com o tempo de secagem em sistemas solar de radiação direta e indireta. Congresso Brasileiro de Ciencia e Tecnologia de Alimentos (CBCTA), Belo Horizonte - MG, v.3, p. 44-

$51,(2008)$.

MACHADO, A.V.; OLIVEIRA, E.L.; SANTOS, E.S.; OLIVEIRA, J.A. Estudo cinético da secagem do pedúnculo de caju e um secador convencional. Revista Verde de Agroecologia e Desenvolvimento Sustentável, Mossoró - RN, v.6, p. 44-51, (2011).

MAIA, J. D. G.; CAMARGO, U. A.; NACHTIGAL, J. C. Avaliação da cv. Isabel em três sistemas de condução e em dois porta-enxertos, para a produção de suco em região tropical. In: CONGRESSO BRASILEIRO DE FRUTICULTURA, 17., 18 a 22 nov. 2002, Belém, PA. Anais... Belém: SBF, 2002. 1 CD-ROM.

MALACRIDA, C. R.; MOTTA, S. Antocianinas em suco de uva: Composição e Estabilidade. B.CEPPA, Curitiba, v. 24, n. 1, p. 59-82 jan./jun. 2006.

MATTIVI, F.; GUZZON, R.; VRHOVSEK, U.; SEFANINI, M.; VELASCO, R. Metabolite profiling of grape: flavonols and anthocyanins. Journal of Agriculture and Food Chemistry, [S.1.], 2006. In press.

MAZZA, G. Anthocyanins in grapes and grape products. Critical Review of Food Science and Nutrition, Madison, v. 35, p. 341-371, 1995.

MUÑOZ-ESPADA, A. C.; WOOD, K. V.; BORDELON, B.; WATKINS, B. A. Anthocyanin quantification and radical scavenging capacity of Concord, Norton, and Marechal Foch grapes and wines. Journal of Agriculture and Food Chemistry, Easton, v. 52, n. 22, p. 6779-6786, 2004.

MELO, P.S.; BERGAMASCHI, K. B.; TIVERON, A. P.; MASSARIOLI, A. P.; OLDONI, T. L. C.; ZANUS, M. C.; PEREIRA, G. E.; ALENCAR, S. M. de. Composição fenólica e atividade antioxidante de resíduos agroindustriais. Ciencia Rural. vol.41 n.6, Santa Maria Junho, 2011.

SANTANA, M. T. A.; SIQUEIRA, H. H.; LACERDA, R. J.; LIMA, L. C. O. Caracterização físico-química e enzimática de uva 'Patricia' cultivada na região de Primavera do Leste - MT. Ciênc. agrotec. vol.32 no.1 Lavras Jan./Feb. 2008. 